\title{
Dimensional Information-Theoretic Measurement of Facial Emotion Expressions in Schizophrenia
}

\author{
Jihun Hamm, ${ }^{1}$ Amy Pinkham, ${ }^{2}$ Ruben C. Gur, ${ }^{3,4}$ Ragini Verma, ${ }^{5}$ and Christian G. Kohler ${ }^{3}$ \\ ${ }^{1}$ Department of Computer Science and Engineering, The Ohio State University, Columbus, OH 43210, USA \\ ${ }^{2}$ Southern Methodist University, Dallas, TX 75205, USA \\ ${ }^{3}$ Department of Psychiatry, Neuropsychiatry Section, University of Pennsylvania, Philadelphia, PA 19104, USA \\ ${ }^{4}$ Philadelphia Veterans Administration Medical Center, Philadelphia, PA 19104, USA \\ ${ }^{5}$ Department of Radiology, Section of Biomedical Image Analysis, University of Pennsylvania, Philadelphia, PA 19104, USA
}

Correspondence should be addressed to Christian G. Kohler; kohler@upenn.edu

Received 9 July 2013; Revised 26 December 2013; Accepted 28 December 2013; Published 25 February 2014

Academic Editor: Robin Emsley

Copyright (c) 2014 Jihun Hamm et al. This is an open access article distributed under the Creative Commons Attribution License, which permits unrestricted use, distribution, and reproduction in any medium, provided the original work is properly cited.

\begin{abstract}
Altered facial expressions of emotions are characteristic impairments in schizophrenia. Ratings of affect have traditionally been limited to clinical rating scales and facial muscle movement analysis, which require extensive training and have limitations based on methodology and ecological validity. To improve reliable assessment of dynamic facial expression changes, we have developed automated measurements of facial emotion expressions based on information-theoretic measures of expressivity of ambiguity and distinctiveness of facial expressions. These measures were examined in matched groups of persons with schizophrenia $(n=28)$ and healthy controls $(n=26)$ who underwent video acquisition to assess expressivity of basic emotions (happiness, sadness, anger, fear, and disgust) in evoked conditions. Persons with schizophrenia scored higher on ambiguity, the measure of conditional entropy within the expression of a single emotion, and they scored lower on distinctiveness, the measure of mutual information across expressions of different emotions. The automated measures compared favorably with observer-based ratings. This method can be applied for delineating dynamic emotional expressivity in healthy and clinical populations.
\end{abstract}

\section{Introduction}

The ability to communicate emotions through facial expressions is crucial for interpersonal engagement. Altered facial expressions of emotions are viewed as characteristic impairments in schizophrenia $[1,2]$ that already feature prominently during early illness $[3,4]$ and may precede the onset of psychosis [5]. Assessment of facial expressions has traditionally relied on observer-based rating scales. The Scale for the Assessment of Negative Symptoms (SANS [2]) and the Positive and Negative Syndrome Scale (PANSS [6]) are commonly used by clinicians to measure facial expressions during interview settings, including the ratings of affective flattening and inappropriate affect. The limited range of quantitative scores obtained during clinical interviews that preferentially focus on positive symptoms without standard prompts to elicit changes in expressivity decrease the ecological validity of facial expression ratings.

An alternative line of investigation of facial expressions of emotions has moved away from clinical assessment of expressions and examined local facial changes based on muscle movements or qualitative assessment of dynamic changes associated with facial expressions. The Facial Action Coding System (FACS $[7,8]$ ) provides detailed descriptions of individual facial muscle movements in multiple facial regions. FACS and some of its adaptations have been applied to schizophrenia [9-13]. However, the FACS procedure requires extensive training and is time-consuming, which makes it difficult to perform in large-scale studies. Due to the prohibitive amount of time to rate videos, the 
FACS procedure is usually performed for static emotional displays. The Facial Expression Coding System (FACES [14]) can classify dynamic facial expressions with details such as valence, type, and intensity of emotions as determined by a rater. FACES ratings of videos of evoked emotions in schizophrenia have shown significant flatness and inappropriateness during spontaneous expressions [15, 16], as well as for simulated [17] and evoked expressions [18].

Media for capturing facial expressions have included still photographs [18-20], videotapes [9-13, 15, 17, 21, 22], and electromyographic recordings [23-25]. Videotaped acquisition offers the advantage of capturing duration and frequency of emotion expressions. However, analysis of such lengthy data sets has been limited to more global assessment of positive and negative emotion expressions, rather than changes in specific face regions. Common measurements of emotion expressions have included recognition rates of expressions [19, 21, 26-28] and FACS-derived measures without analysis of specific AUs [9-12, 15, 17]. Other methods have included computerized face morphometry [25, 27] and electromyographic measurements [23-25, 29, 30] that can measure minute muscle activations, albeit limited to select face regions, for example, zygomatic and corrugator muscles that are typically utilized in frowning and smiling expressions. An interesting and frequent finding arising from these investigations has been the possible disconnectedness between emotion expressions and subjective emotion experience $[18,30,31]$, which in turn may affect social interactions in schizophrenia.

In an effort to derive more objective and scalable measurement of facial expressivity, we have pursued computerized measurement of facial expressions that were obtained during standardized procedures to elicit emotion expressions. Initial efforts based on manually defined face regions from still images in healthy actors [32] and persons with schizophrenia [33] showed significant group differences in expressions of happiness, sadness, anger, and fear. Wang et al. [34] refined the method to allow automated analysis of videos and demonstrated different frequencies of four emotions in three exemplar participants. To account for low intensity and ambiguous expressions of emotions, Hamm et al. [35] proposed automated measurement of individual facial muscles using FACS parameters and demonstrated the differences of facial muscle activities in four patients and controls. However, the implications of altered individual facial muscle movements on global expressivity and respective differences in schizophrenia, where facial expressions are commonly impaired, remain to be better elucidated. In this paper, we present novel measures of facial expressivity, based on individual muscle movements that are derived from information theory [36] and applied these measures to matched groups of schizophrenia and control subjects. Information theory originated in the 1950s from the field of applied mathematics to analyze the capacity of communication channels. The method has provided powerful tools to analyze human performance measures in psychological experiments [37] and a framework for understanding emotional communications [38]. Information theory has also been successfully used in biomedical investigations, such as processing capacity of neural coding [39], complexity in electrocardiogram sequences [40], and electroencephalography in Alzheimer's disease [41] and schizophrenia [42].

As an extension of our previous work that examined both observer-based and computerized measurements of facial expressivity of emotions, we tested sensitivity of the information-theoretic measures in characterizing and quantifying affective expression deficits in schizophrenia, and we examined their relationship with observer-based ratings of inappropriate and flattened emotion expressions. We applied two-dimensional measures of facial expressivity that can be computed objectively from videos of facial expressions without requiring observer-based ratings: (1) ambiguity of facial expressions within a single emotion and (2) distinctiveness of facial expressions across separate emotions. These measures correspond to the two most important information-theoretic quantities of (1) conditional entropy and (2) mutual information. Briefly, ambiguity is the amount of uncertainty in a person's facial muscle patterns during expression of a single emotion as contrasted with consistency of the pattern. A person whose facial muscle pattern is only brief or is variable during emotion expression will be less effective in communicating his or her intended emotion to another person. Distinctiveness is the capacity of a person to express different emotions succinctly through facial muscles. A person who is unable to produce distinct facial patterns for different emotions will also be less effective in communicating a specific emotion. We anticipated that ambiguity and distinctiveness measures can be applied to large data sets of dynamic expressions and they can capture aspects of expressivity deficits that would improve our understanding of emotion expression abilities in persons with schizophrenia. In addition, although representing different theoretical constructs, we examined whether informationtheoretic measures correlate with observer-based ratings such as inappropriate and flattened affect.

\section{Methods}

2.1. Subjects. We collected videos of healthy controls and persons with schizophrenia for a neuropsychiatric study of emotions under an approved IRB protocol at the University of Pennsylvania. After describing the study to the subjects, we obtained written informed consents, including consent to publish pictures. There were 28 outpatients with a DSM-IV diagnosis of schizophrenia and 26 healthy controls balanced in gender, race, age, and parental education. All patients were clinically stable without hospitalization for at least 3 months prior to research assessment and had been maintained on their present medication for the past month. Presence of significant acute extrapyramidal symptoms as evidenced by a score of 1 or higher on at least 2 of the rigidity or tremor items (items 2, 3, 4, 5, and 9) was exclusionary. Likewise presence of significant tardive extrapyramidal symptoms as evidenced by a score of 2 or higher on items 1-4 (facial and oral movements) was exclusionary. All patients were treated 
TABLE 1: Demographic and clinical characteristics.

\begin{tabular}{lcc}
\hline & Patient group & Control group \\
\hline Gender (M:F) & $14: 14$ & $16: 10$ \\
Ethnicity & & \\
$\quad$ (Caucasian : African-American : other) & $9: 16: 3$ & \\
Age & & $3: 15: 3$ \\
$\quad$ Mean & $32.3 \pm 9.0$ & $33.0 \pm 8.6$ \\
$\quad$ Range & $20-47 \mathrm{yrs}$ & $20-48$ yrs \\
Duration of illness & $13.00 \pm 8.3$ & N/A \\
$\quad$ Range & $2-33$ & N/A \\
Symptoms & & N/A \\
$\quad$ Positive (SAPS-total) & $6.00 \pm 8.5$ & N/A \\
$\quad$ Negative (SANS-total) & $13.00 \pm 8.3$ & N/A \\
Antipsychotics & & N/A \\
$\quad$ Chlorpromazine-equivalents $(n=28)$ & $225 \mathrm{mg} \pm 247$ & $13.6 \mathrm{mg} \pm 7.6$ \\
$\quad$ Olanzapine-equivalents $(n=2)$ & & \\
\end{tabular}

Image/video

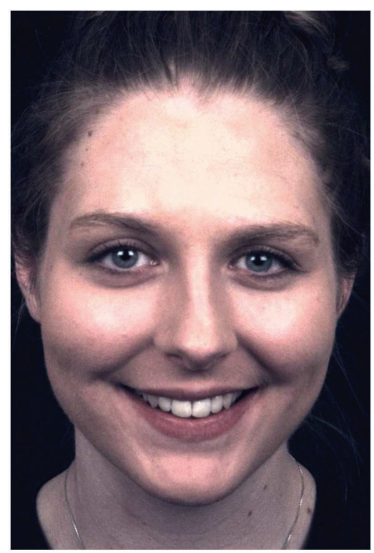

Feature extraction

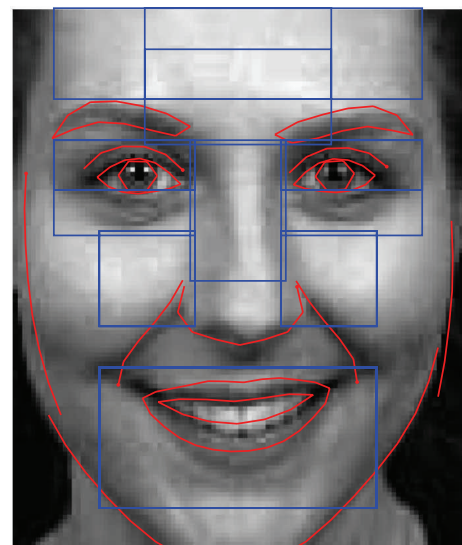

Action unit likelihoods

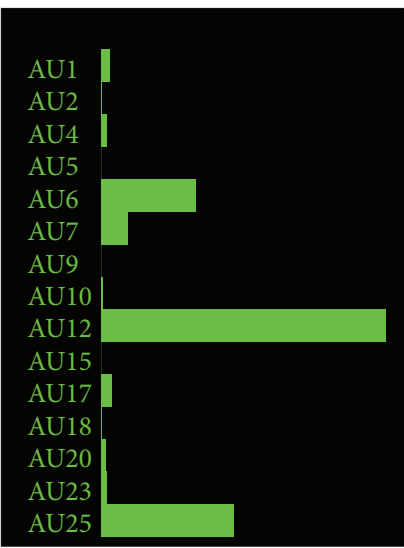

FIgURE 1: Automated Action Unit recognition system. For each frame of a video, geometric changes in facial components (red curves) are automatically tracked, and textural changes due to temporary wrinkles are detected in multiple regions of interest (blue boxes). These extracted features are fed through pattern classifiers to yield activity of each Action Unit (green bars: longer bar means higher activity).

with second-generation antipsychotics that were converted to dosages equivalent to olanzapine (OLZ); two patients were also treated with first-generation antipsychotics that were converted to dosages equivalent to chlorpromazine (CPZ). All medication dosages were stable for the past month prior to testing and no patient was treated with anticholinergic medications. Pertinent demographic and clinical information is summarized in Table 1.

\subsection{Emotion Elicitation Procedure and Video Acquisition. To} test emotion expression ability, we followed the emotion elicitation procedure previously described [43] and adapted it for use in schizophrenia [13]. Videos were obtained for neutral expressions and for five universal emotions (happiness, sadness, anger, fear, and disgust). Before recording, participants were asked to describe biographical emotional situations, when each emotion was experienced in mild, moderate, and high intensities, and these situations were summarized as vignettes. Subsequently, subjects were seated in a brightly lit room where recordings took place, and emotional vignettes were recounted to participants in a narrative manner using exact wording derived from the vignettes. The spontaneous facial expressions of the subjects were recorded as videos. Before and between the five emotion sessions, the subjects were asked to relax and return to a neutral state. The duration of each session was about two minutes (110 $\pm 54 \mathrm{sec})$.

2.3. Video Processing and Automated FACS. Our group has developed an automated facial coding procedure that can score intensity of facial muscle activity known as Action Units (AUs $[7,8]$ ) by computerized analysis of videos. The details of the system and the validation of accuracy appeared in Hamm et al. [35], and here we describe key components of the system and how we used it to measure information (see Figure 1). Videos acquired in evoked emotions were analyzed frame by frame. For each frame, geometric changes in facial 
components such as eyes, eyebrows, nasolabial line, and lips were automatically tracked, and textural changes due to temporary wrinkles were detected in multiple regions of the face. To increase the reliability of the tracking, a small fraction $(\sim 3 \%)$ of frames from videos in which the face leaves the screen (i.e., nonfrontal) were automatically discarded from analysis. Extracted geometric and texture features were then passed through pattern classifiers to yield intensity of the following 15 AUs: AU1 (Inner Brow Raiser), AU2 (Outer Brow Raiser), AU4 (Brow Lowerer), AU5 (Upper Lid Raiser), AU6 (Cheek Raise), AU7 (Lid Tightener), AU9 (Nose Wrinkler), AU10 (Upper Lip Raiser), AU12 (Lip Corner Puller), AU15 (Lip Corner Depressor), AU17 (Chin Raiser), AU18 (Lip Puckerer), AU20 (Lip Stretcher), AU23 (Lip Tightener) and AU25-27 (Lips Part and Mouth Open). The other AUs than these 15 AUs were not used since they were too infrequently in the recorded videos.

2.4. Computation of Ambiguity and Distinctiveness of Facial Expressions. From the distribution of 15-dimensional continuous variables collected from videos, we computed information-theoretic measures of expressivity for each subject. Specifically, we measure ambiguity of facial expression patterns within each emotion and distinctiveness of facial expression patterns across emotions. These two measures correspond to conditional entropy and mutual information, which are the fundamental variables of information theory [36]. Interpretations of the two information-theoretic measures depend on the experiments being conducted, and ambiguity and distinctiveness are specific interpretations of our experimental results with the spontaneous expression of emotions. In psychology, the two measures have been called equivocation and information transmission, respectively, in the context of absolute judgment tasks [44].

Computation of ambiguity and distinctiveness requires estimation of differential entropy from facial muscles. Differential entropy is the amount of uncertainty in continuous probabilistic distributions from which we derive mutual information and conditional entropy. Let $x$ denote the (discrete) emotional state of an individual and $y$ denote the (continuous and multivariate) facial muscle activity. Differential entropy is then defined by $H(p(y))=E[-\log p(y)]=$ $-\int_{\mathbb{R}^{d}} p(y) \log p(y) d y$. Unlike discrete entropy, differential entropy is a relative measure and can have negative values; a univariate Gaussian distribution with $\sigma=(2 \pi e)^{-1 / 2}$ has zero differential entropy, and Gaussians with narrower or wider peaks have negative or positive entropy, respectively. Since we do not know $p(y)$ a priori, the differential entropy has to be estimated from samples, much like estimation of mean and variance. Several estimation methods have been proposed, including adaptive partitioning, kernel density estimation, and nearest neighbor estimation (see Beirlant et al. [45] for a review). We used a $k$-nearest neighbor estimator of entropy [46-48].

$H(p(y)) \approx(d / n) \sum_{i}^{n} \log \rho+v_{d}-\psi(k)+\log n$. The $v_{d}=$ $\pi^{d / 2} / \Gamma(d / 2+1)$ is the volume of a $d$-dimensional unit sphere, where $\Gamma$ is the gamma function $\Gamma(z)=\int_{0}^{\infty} t^{z-1} e^{-t} d t$ and $\psi(z)$ is the digamma function $\psi(z)=\Gamma(z)^{\prime} / \Gamma(z)$. The only free parameter of nearest-neighbor estimate is the size of the neighbors $k$ for which we used the heuristic rule [47].

$k=$ round $\left(n^{1 / 2}+0.5\right)$, where $n$ is the number of samples. For numerical stability, we also added negligible amount (= $10^{-8}$ ) of random noise to the data while computing entropy.

In our experiments, the conditional entropy is defined as $H(y \mid x)=\sum_{x=x_{i}} p\left(x_{i}\right) H\left(y \mid x_{i}\right)=\sum_{x=x_{i}} p\left(x_{i}\right) \int-p(y \mid$ $\left.x_{i}\right) \log \left(p\left(y \mid x_{i}\right)\right) d y$, which is the average entropy of facial expression per emotion computed from facial muscle activity $y$ in the video of each emotion $x$ with equal priors for each emotion $(p(x)=1 / 5)$. We refer to this conditional entropy as ambiguity in the following context: when an individual's facial expression is consistent within each emotion, the conditional entropy is low, and when the expression is varying and ambiguous within each emotion, the conditional entropy is high.

The mutual information $I(x, y)$ can be computed from $I(x ; y)=H(y)-H(y \mid x)$. Mutual information between discrete and continuous variables, as in our case, is also known as Jensen-Shannon divergence $[49,50]$ and is nonnegative and bounded. By reformulating $I(x ; y)$ as the average KLdivergence [51] between conditional $p(y \mid x)$ and marginal $p(y)$ distributions $I(x ; y)=K L(p(x, y) \| p(x) p(y))=$ $\sum_{x} p(x) \int p(y \mid x) \log (p(y x) / p(y)) d y=\sum_{x} p(x) K L(p(y \mid$ $x) \| p(y))$, we notice that mutual information measures the average distance of emotion-specific facial expression pattern $(p(x \mid y))$ from the patterns of all emotions combined $(p(x))$. Hence our choice of the term distinctiveness is for mutual information.

2.5. Interpretation of Ambiguity and Distinctiveness Measures. In this paper, we report the ambiguity and the distinctiveness as $z$-scores $(=(x-m) / s)$ instead of their raw values for an easier interpretation, where the mean and the standard deviation are computed using all subjects and conditions. We do this because these values are unitless and dependent on the experimental setting and therefore meaningful only in a relative sense. For example, if we use six basic emotions including "surprise" instead of five, the absolute values of ambiguity and distinctiveness will change. However, the difference of values in diagnostic groups or conditions still measures the relative amount of the ambiguity and distinctiveness of facial expressions and provides meaningful information. Note that the standardization of raw values using $z$-scores does not affect the subsequent statistical analysis.

Lastly, the ambiguity and the distinctiveness are computed across all emotions and not for individual emotions. While it is possible to analyze ambiguity for each emotion, pooling the values across emotions results in more reliable measures.

\subsection{Observer-Based Measures}

Validation of Ambiguity and Distinctiveness. To verify that the information-theoretic measures agree with an observer's interpretation of ambiguity and distinctiveness from videos, 
the following criteria for manual scores from 0 to 4 were defined, and each video was rated by an observer blind to the diagnosis of subjects. For ambiguity, which was rated for videos of each emotion, 0 meant very consistent (with only single facial expression pattern in the single video of an emotion) and 4 meant very ambiguous (with more than four different facial expression patterns in the video of an emotion). Scores 1 to 3 corresponded to intermediate levels of ambiguity (with 1, 2, and 3 major facial expression patterns in a video, resp.). For distinctiveness, which was rated across videos of five emotions of a single subject, 0 meant the five emotional videos were totally indistinguishable in representing the target emotions, and 4 meant all five videos were distinctive and representative of the target emotions. Scores 1 to 3 corresponded to intermediate levels of distinctiveness (with 1, 2, and 3 videos of distinctive emotions, resp.).

Observer-Based Ratings of Facial Expressions. To compare information measures with previous observer-based ratings, flatness and inappropriateness of facial expression from the Scale for the Assessment of Negative Symptoms (SANS) were adapted to video-based ratings [33]. Two raters scored each video with separate scores for flat and inappropriate affect, ranging from 0 (none) to 4 (extremely flat or inappropriate). SANS raters knew the intended emotion of the video but not the diagnosis of subjects. Videobased SANS was based on a 5-point rating, similar to observer-based SANS. Ratings that differed by 2 or more points were reviewed for consensus and final ratings were averaged.

2.7. Statistical Analysis. The following data analysis was performed to demonstrate applicability and validity of the measures of ambiguity and distinctiveness. Group-based comparisons for ambiguity and distinctiveness of expressions involved were carried out via two-way ANOVA of ambiguity and distinctiveness separately, using sex and diagnosis as grouping factors. We also measured the effect size of diagnosis by Cohen's $d\left(=\left(m_{1}-m_{2}\right) /\left(0.5\left(s_{1}^{2}+s_{2}^{2}\right)\right)^{1 / 2}\right)$. Validation of computerized measures of ambiguity and distinctiveness against observer-based measures was performed by Pearson correlations, where higher values meant better agreement between the computerized and observer-based measures. For observer-based ratings of inappropriateness and flatness of expressions we performed separate two-way ANOVA using sex and diagnosis as grouping factors. We also measured the effect size of diagnosis by Cohen's $d$. For the purposes of interpretability and comparison with alternative measures of symptom severity, we performed multiple regression analysis to study the explanatory power of the computerized measures of ambiguity and distinctiveness for observer-based ratings of inappropriate and flat affects, in which computerized measures were used as independent variables and each of the observer-based ratings was used as a dependent variable. We performed multiple regression also in the other direction, in which observer-based ratings were used as independent variables and each of the computerized measures was used as a dependent variable.

\section{Results}

3.1. Ambiguity and Distinctiveness of Emotion Expressions. Ambiguity of expression, averaged across emotions, showed a strong effect of diagnosis $(F=12, d f=1,53, P=0.0012)$, but no effect of sex $(F<0.001, d f=1,53, P=0.99)$ nor interaction $(F=0.78, d f=1,53, P=0.38)$ by twoway ANOVA. Patients showed higher ambiguity $(0.41 \pm 1.0)$ than controls $(-0.44 \pm 0.79)$, with a large effect size of 0.93 (Cohen's $d$ ). Likewise, distinctiveness of expression showed a strong effect of diagnosis $(F=8.3, d f=1,53, P=0.0057)$, but no effect of sex $(F=0.056, d f=1,53, P=0.81)$ nor interaction $(F=0.26, d f=1,53, P=0.61)$ by two-way ANOVA. Patients showed lower distinctiveness $(-0.37 \pm 0.88)$ than controls $(0.40 \pm 0.98)$ with a large effect size of Cohen's $d=0.83$.

The characteristics of ambiguity and distinctiveness measures are demonstrated with sample videos of subjects in Figures 2-5 that illustrate the expressions of subjects with ratings for low ambiguity/high distinctiveness, in contrast to subjects with ratings for low ambiguity/low distinctiveness, high ambiguity/low distinctiveness, and high ambiguity/high distinctiveness.

\subsection{Observer-Basted Measures}

Validation of Ambiguity and Distinctiveness. The computerized measures of ambiguity and distinctiveness were well correlated with the observer's scores of ambiguity $(r=0.71, P<$ $0.01)$ and distinctiveness $(r=0.60, P<0.01)$. These correlations supported the notion of agreement between the computerized measures from information theory and the observer-rated measures from visual examination of the videos.

Observer-Based Ratings of Facial Expressions. For videobased expert SANS ratings, flatness of expression showed a moderate effect of diagnosis $(F=4.6, d f=1,53, P=$ $0.038)$, but no effect of sex $(F=3.9, d f=1,53, P=$ $0.055)$ nor interaction $(F=0.19, d f=1,53, P=0.66)$ by two-way ANOVA. Patients were more flat $(2.0 \pm 0.93)$ than controls $(1.5 \pm 0.91)$, with a medium effect size of 0.57 . The inappropriateness of expression showed a strong effect of diagnosis $(F=9.6, d f=1,53, P=0.0033)$, but no effect of sex $(F=0.99, d f=1,53, P=0.33)$ nor interaction $(F=0.0047, d f=1,53, P=0.95)$ by two-way ANOVA. Patients were rated higher on inappropriate affect $(1.1 \pm 0.65)$ than controls $(0.63 \pm 0.37)$ with a large effect size of 0.92 .

\subsection{Relationship between Computerized and Observer-} Based Measures. We examined the relationships between information-theoretic measures of ambiguity and distinctiveness and observer-based measures of inappropriate 
and flattened facial expressions of emotions. Regression of ambiguity on observer-based measures was moderately predictive $\left(R^{2}=0.13, F=3.4, P=0.040\right)$, and the coefficients from flatness and inappropriateness were -0.14 $(P=0.30)$ and $0.50(P=0.032)$, respectively. Regression of distinctiveness on observer-based measures was highly predictive $\left(R^{2}=0.19, F=5.8, P=0.0056\right)$, and the coefficients from flatness and inappropriateness were -0.32 $(P=0.027)$ and $-0.65(P=0.0063)$, respectively. Regression of flatness on computerized measures was moderately predictive $\left(R^{2}=0.15, F=4.3, P=0.019\right)$, and the coefficients from ambiguity and distinctiveness were -0.33 $(P=0.025)$ and $-0.34(P=0.013)$ respectively. Lastly, regression of inappropriateness on computerized measures was moderately predictive $\left(R^{2}=0.15, F=4.3, P=0.018\right)$, and the coefficients from ambiguity and distinctiveness were $0.14(P=0.11)$ and $-0.14(P=0.11)$, respectively.

\section{Discussion and Conclusions}

Impaired abilities of facial expression of emotions are common dysfunctions in schizophrenia that are associated with worse quality of life and poorer outcome [52, 53]. Clinical assessment of emotion expression abilities is typically obtained using observer-based rating scales administered during interviews that are not standardized to elicit emotion expressions and with limited potential to compare data across different studies and populations. More advanced observer-based measurements of facial expressions have been challenged by the complexity inherent in rating regional and global changes in dynamic facial expressions and their applications have been mainly limited to research. Nevertheless these investigations using standardized observer based ratings and electromyography have underscored that persons with schizophrenia exhibit altered expressions of volitional and of more fleeting spontaneous facial emotions that do not necessarily reflect their internal emotional state. Another challenge of measuring emotion expressions has been the importance to obtain emotion expressions that are genuine and naturalistic and not obscured by the artifice of the testing setting and methodology or influenced by rater fatigue. This is where automated computerized measurement offers an advantage over other methods to investigate dynamic expressions.

Our group has developed computerized assessment of emotion expression abilities that allows for objective measurement of facial expressions that are obtained in a standardized setting [32-35]. As an extension to these efforts, we have applied computerized assessments of facial expressivity to patients with schizophrenia and healthy controls to examine whether the novel methodology can elucidate differences in facial expression of emotions. Computerized informationtheoretic measures focus on differences in ambiguity, which represents the measure of variability within the expression of a single emotion, and distinctiveness, which represents the measure of how distinctive facial expressions are for a particular emotion in comparison to other emotions. The computerized approach was validated by observer-based ratings of ambiguity and distinctiveness. In addition when computerized measures were predicted by observer-based ratings, ambiguity was positively related to inappropriateness, and distinctiveness was negatively related to both flatness and inappropriateness. Conversely, when observer-based ratings were predicted by computerized measures, flatness was negatively related to both ambiguity and distinctiveness, while inappropriateness was not significantly related with either measure alone, although it was significantly predicted by overall ambiguity and distinctiveness. As illustrated in Figures 2-5, computerized measures of ambiguity and distinctiveness were associated with facial expressions that, in the combination of low ambiguity/high distinctiveness (Figure 2), were well recognizable within each emotion and also different across emotions. High ambiguity with either low (Figure 3) or high (Figure 4) distinctiveness values appeared as inappropriate expressions that were either similar or different across emotions. The computerized measures also indicate flatness, as when facial expressions are not variable within an emotion (low ambiguity) and also indistinguishable across emotions (low distinctiveness) (Figure 5). These results suggest that the information-theoretic measures of emotion expressions are related to the observerbased ratings and computerized measures may provide quantifiable information on different causes of observed inappropriate affect. Specifically, inappropriateness of expression may result either from ambiguous and inconsistent facial expressions in each emotion regardless of distinctiveness across emotions. Flat affect, on the other hand, was mainly related to emotion expressions being indistinct across emotions.

Our computerized method employing informationtheoretic measures offers several methodological advantages over currently available observer-based rating scales and FACS-based rating instruments, for example, our method being more objective and repeatable for any number of subjects, at the same time being less labor intensive and timeconsuming. It is more objective than FACES since the method does not involve observer-judgment of emotions expressed and is less labor/time intensive than FACS and FACES since it is fully automated similar to EMG, without the inconvenience of physically placing electrodes on the participants' faces.

While we demonstrated the feasibility and applicability of these measures with schizophrenia patients and healthy controls, we are applying the analysis to a larger sample that can better reflect the range of individual differences in the respective populations. A major limitation of our approach is based on the fact that no computerized determination was made regarding emotional valence and such qualitative information could have enhanced the results based on information-theoretic measures of expressivity. Another limitation of the current study is that the induction procedure within the laboratory, while standardized, may lack ecological 


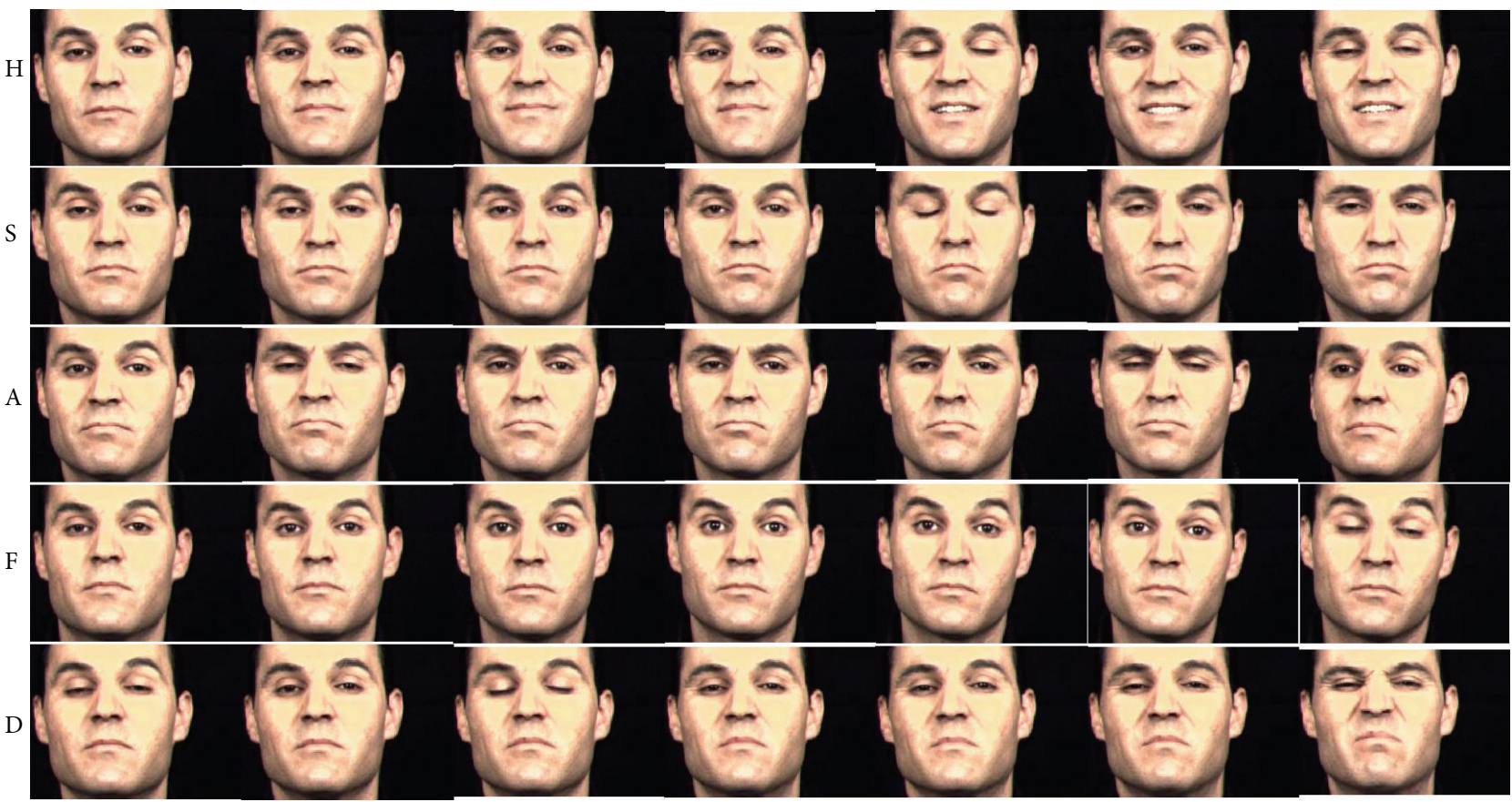

Figure 2: Sample videos of a subject with low ambiguity and high distinctiveness. H, S, A, F, and D stand for happiness, sadness, anger, fear, and disgust. Each row shows sample images evenly chosen from a video of the emotion. Facial expressions of low ambiguity and high distinctiveness are consistent within each emotion and also different across emotions, which makes each emotion well recognizable.

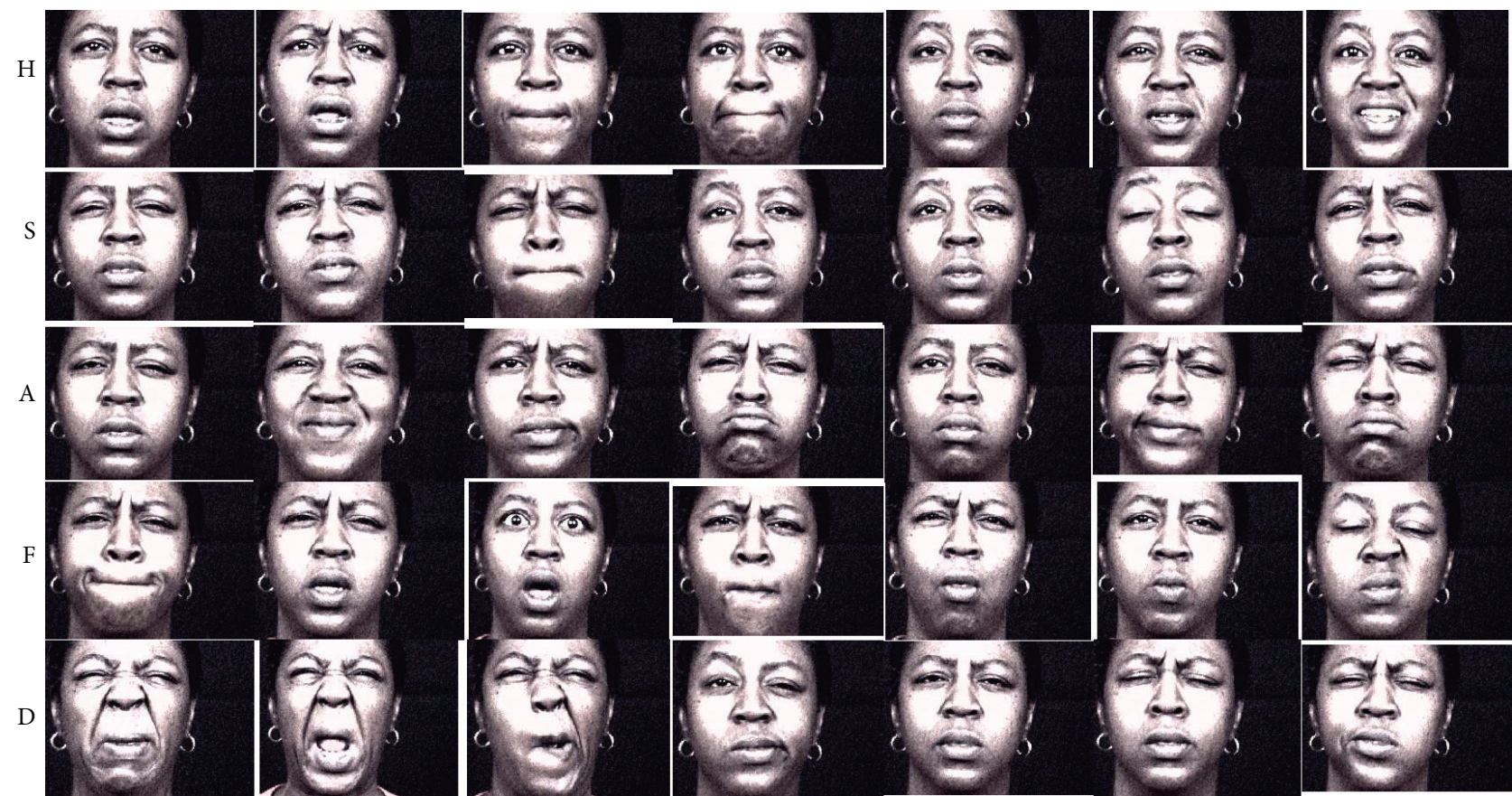

Figure 3: Sample videos of a subject with high ambiguity and low distinctiveness. High ambiguity and low distinctiveness appear as inappropriate facial expressions, due to the lack of consistent patterns within each emotion and the overlap of patterns across emotions.

validity. A more naturalistic setting would include filming participants while they recount their own experiences. Unfortunately at the present state of this methodology, facial movements on account of speech would generate too much noise for our algorithms to overcome.
In conclusion, our method offers an automated means for quantifying individual differences in emotional expressivity, similar to what has been accomplished in the area of emotion recognition. This capability opens new venues for delineating emotional expressivity in healthy people and across clinical 

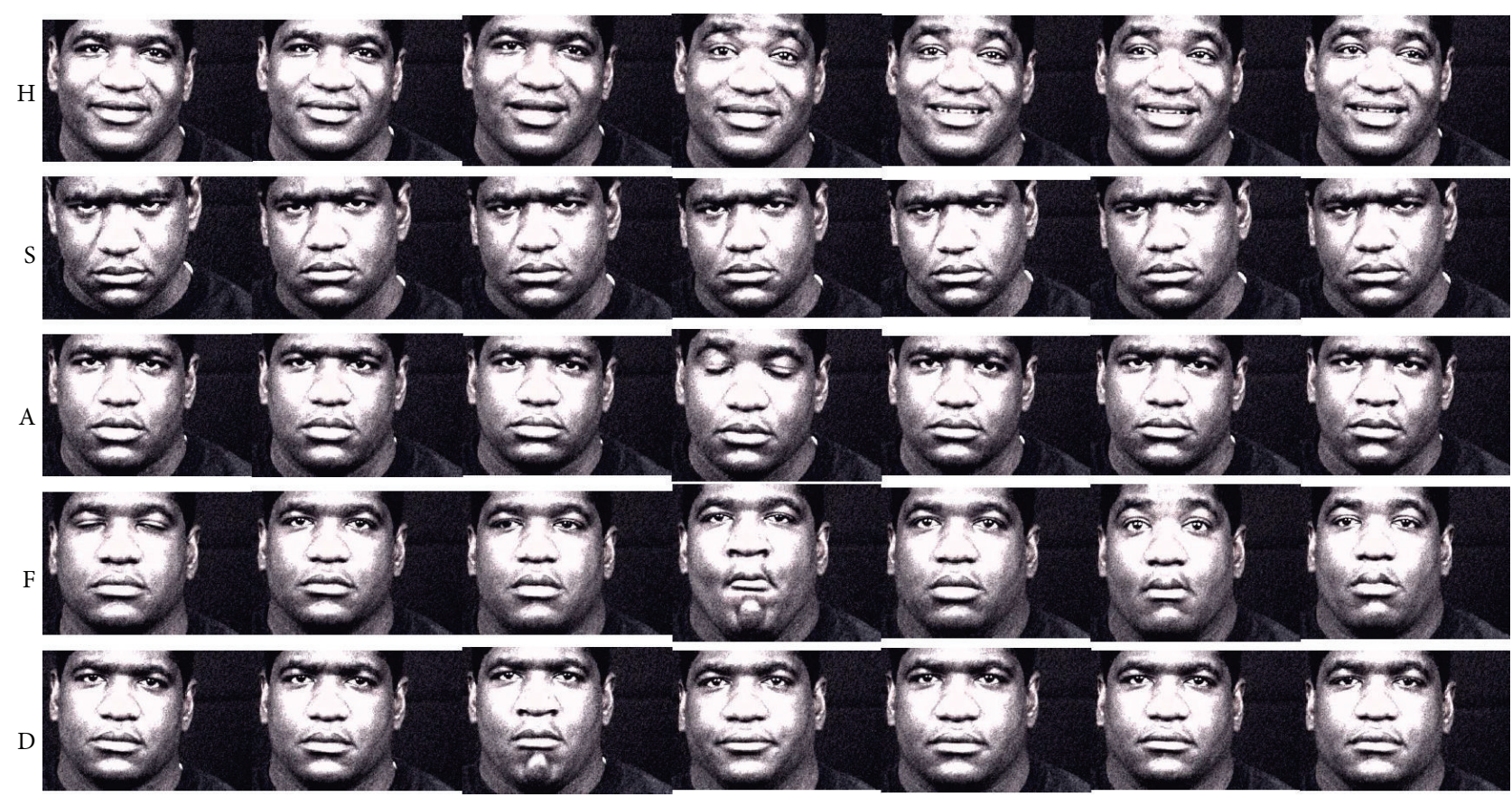

FIGURE 4: Sample videos of a subject with high ambiguity and high distinctiveness. High ambiguity and high distinctiveness also appear as inappropriate facial expressions due to the lack of consistent patterns within each emotion.

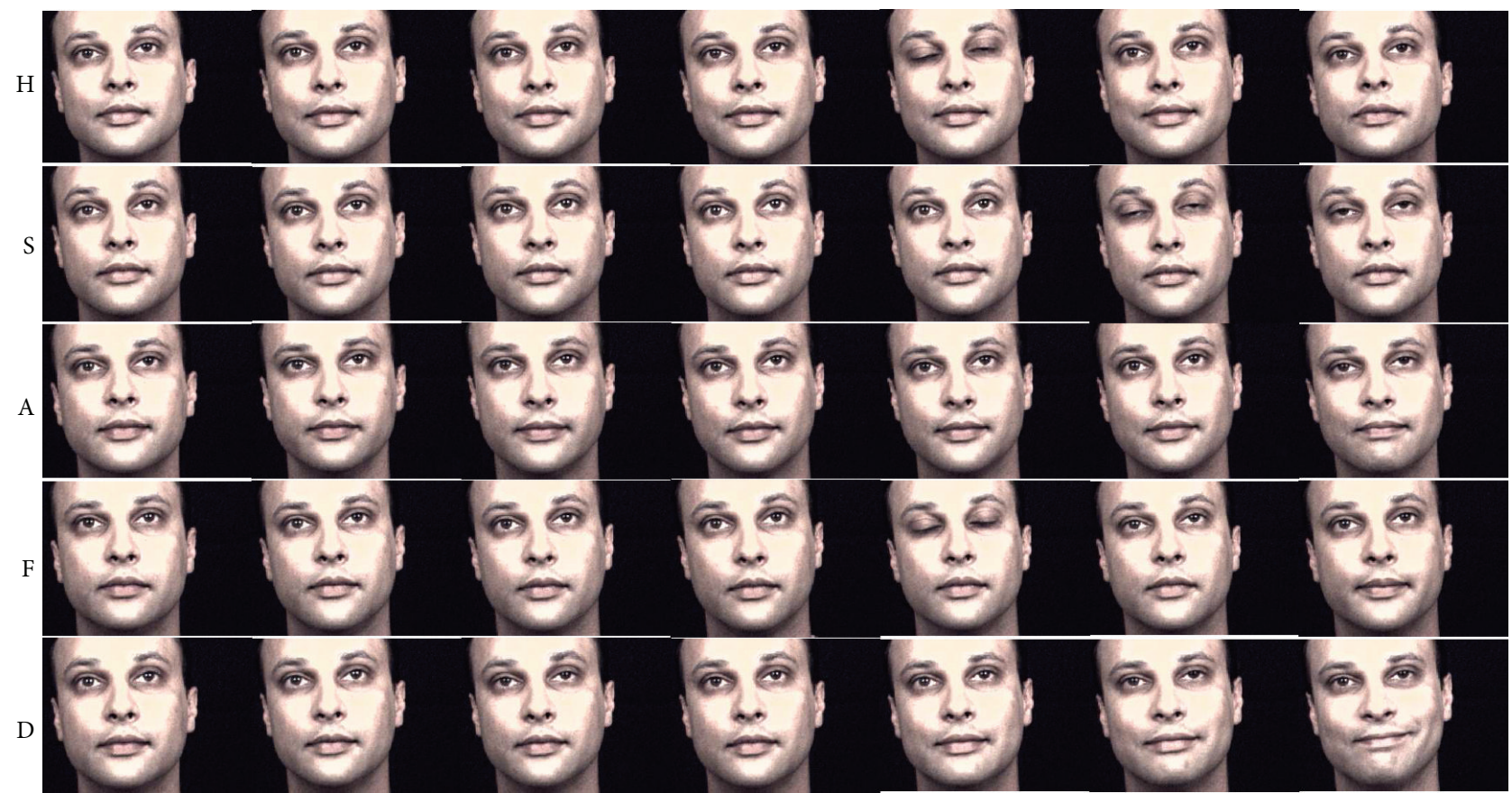

Figure 5: Sample videos of a subject with low ambiguity and low distinctiveness. Facial expressions of low ambiguity and low distinctiveness present themselves as muted or flat expressions throughout all emotions.

populations. As for investigations in healthy population, the automated procedure is well suited to examine both age and gender related changes in emotion expression abilities. Potential clinical applications may include repeated monitoring of facial expressions to investigate effects of disease progression and more general treatment effects or to measure targeted efforts to remediate emotion expression abilities. Automated comparisons need not be limited to select clinical populations and may allow for effective comparisons of emotion expressivity across different psychiatric disorders. 


\section{Conflict of Interests}

The authors declare that there is no conflict of interests regarding the publication of this paper.

\section{Acknowledgments}

This work was supported by the National Institutes of Health (R01-MH073174 and R01-MH060722) and by Stanley Foundation Grant. The authors thank Elizabeth Martin and Kristin Healey for their assistance in data acquisition.

\section{References}

[1] E. Bleuler, "Dementia praecox oder die gruppe der schizophrenien," 1911.

[2] N. C. Andreasen, The Scale for the Assessment of Negative Symptoms (SANS). The Scale for the Assessment of Positive Symptoms (SAPS), The University of Iowa, Iowa City, Iowa, USA, 1984.

[3] A. K. Malla, J. J. Takhar, R. M. G. Norman et al., "Negative symptoms in first episode non-affective psychosis," Acta Psychiatrica Scandinavica, vol. 105, no. 6, pp. 431-439, 2002.

[4] J. Mäkinen, J. Miettunen, M. Isohanni, and H. Koponen, "Negative symptoms in schizophrenia-a review," Nordic Journal of Psychiatry, vol. 62, no. 5, pp. 334-341, 2008.

[5] E. F. Walker, K. E. Grimes, D. M. Davis, and A. J. Smith, "Childhood precursors of schizophrenia: facial expressions of emotion," American Journal of Psychiatry, vol. 150, no. 11, pp. 1654-1660, 1993.

[6] S. R. Kay, A. Fiszbein, and L. A. Opler, "The positive and negative syndrome scale (PANSS) for schizophrenia," Schizophrenia Bulletin, vol. 13, no. 2, pp. 261-276, 1987.

[7] P. Ekman and W. Friesen, Facial Action Coding System: Investigators Guide, Consulting Psychologists Press, Palo Alto, Calif, USA, 1978.

[8] P. Ekman and W. Friesen, Manual of the Facial Action Coding System (FACS), Consulting Psychologists Press, Palo Alto, Calif, USA, 1978.

[9] H. Berenbaum, "Posed facial expressions of emotion in schizophrenia and depression," Psychological Medicine, vol. 22, no. 4, pp. 929-937, 1992.

[10] H. Berenbaum and T. F. Oltmanns, "Emotional experience and expression in schizophrenia and depression," Journal of Abnormal Psychology, vol. 101, no. 1, pp. 37-44, 1992.

[11] W. Gaebel and W. Wölwer, "Facial expressivity in the course of schizophrenia and depression," European Archives of Psychiatry and Clinical Neuroscience, vol. 254, no. 5, pp. 335-342, 2004.

[12] F. Trémeau, D. Malaspina, F. Duval et al., "Facial expressiveness in patients with schizophrenia compared to depressed patients and nonpatient comparison subjects," American Journal of Psychiatry, vol. 162, no. 1, pp. 92-101, 2005.

[13] C. G. Kohler, E. A. Martin, N. Stolar et al., "Static posed and evoked facial expressions of emotions in schizophrenia," Schizophrenia Research, vol. 105, no. 1-3, pp. 49-60, 2008.

[14] A. M. Kring and D. M. Sloan, "The facial expression coding system (FACES): development, validation, and utility," Psychological Assessment, vol. 19, no. 2, pp. 210-224, 2007.

[15] A. M. Kring, S. L. Kerr, D. A. Smith, and J. M. Neale, "Flat affect in schizophrenia does not reflect diminished subjective experience of emotion," Journal of Abnormal Psychology, vol. 102 , no. 4, pp. 507-517, 1993.

[16] A. M. Kring and J. M. Neale, "Do schizophrenic patients show a disjunctive relationship among expressive, experiential, and psychophysiological components of emotion?" Journal of Abnormal Psychology, vol. 105, no. 2, pp. 249-257, 1996.

[17] M. A. Aghevli, J. J. Blanchard, and W. P. Horan, “The expression and experience of emotion in schizophrenia: a study of social interactions," Psychiatry Research, vol. 119, no. 3, pp. 261-270, 2003.

[18] C. G. Kohler, E. A. Martin, M. Milonova et al., "Dynamic evoked facial expressions of emotions in schizophrenia," Schizophrenia Research, vol. 105, no. 1-3, pp. 30-39, 2008.

[19] E. Gottheil, C. C. Thornton, and R. V. Exline, "Appropriate and background affect in facial displays of emotion. Comparison of normal and schizophrenic males," Archives of General Psychiatry, vol. 33, no. 5, pp. 565-568, 1976.

[20] B. L. Schwartz, J. Mastropaolo, R. B. Rosse, G. Mathis, and S. I. Deutsch, "Imitation of facial expressions in schizophrenia," Psychiatry Research, vol. 145, no. 2-3, pp. 87-94, 2006.

[21] K. M. Putnam and A. M. Kring, "Accuracy and intensity of posed emotional expressions in unmedicated schizophrenia patients: vocal and facial channels," Psychiatry Research, vol. 151, no. 1-2, pp. 67-76, 2007.

[22] E. Steimer-Krause, R. Krause, and G. Wagner, "Interaction regulations used by schizophrenic and psychosomatic patients: studies on facial behavior in dyadic interactions," Psychiatry, vol. 53, no. 3, pp. 209-228, 1990.

[23] K. S. Earnst, A. M. Kring, M. A. Kadar, J. E. Salem, D. A. Shepard, and P. T. Loosen, "Facial expression in schizophrenia," Biological Psychiatry, vol. 40, no. 6, pp. 556-558, 1996.

[24] A. M. Kring, S. L. Kerr, and K. S. Earnst, "Schizophrenic patients show facial reactions to emotional facial expressions," Psychophysiology, vol. 36, no. 2, pp. 186-192, 1999.

[25] R. M. Mattes, F. Schneider, H. Heimann, and N. Birbaumer, "Reduced emotional response of schizophrenic patients in remission during social interaction," Schizophrenia Research, vol. 17, no. 3, pp. 249-255, 1995.

[26] E. Gottheil, A. Paredes, R. V. Exline, and R. Winkelmayer, "Communication of affect in schizophrenia," Archives of General Psychiatry, vol. 22, no. 5, pp. 439-444, 1970.

[27] F. Schneider, H. Heimann, W. Himer, D. Huss, R. Mattes, and B. Adam, "Computer-based analysis of facial action in schizophrenic and depressed patients," European Archives of Psychiatry and Clinical Neuroscience, vol. 240, no. 2, pp. 67-76, 1990.

[28] B. L. Schwartz, J. Mastropaolo, R. B. Rosse, G. Mathis, and S. I. Deutsch, "Imitation of facial expressions in schizophrenia," Psychiatry Research, vol. 145, no. 2-3, pp. 87-94, 2006.

[29] K. Wolf, R. Mass, F. Kiefer, K. Wiedemann, and D. Naber, "Characterization of the facial expression of emotions in schizophrenia patients: preliminary findings with a new electromyography method," Canadian Journal of Psychiatry, vol. 51, no. 6, pp. 335341, 2006.

[30] M. Iwase, K. Yamashita, K. Takahashi et al., "Diminished facial expression despite the existence of pleasant emotional experience in schizophrenia," Methods and Findings in Experimental and Clinical Pharmacology, vol. 21, no. 3, pp. 189-194, 1999.

[31] C. E. Sison, M. Alpert, R. Fudge, and R. M. Stern, "Constricted expressiveness and psychophysiological reactivity in schizophrenia," Journal of Nervous \& Mental Disease, vol. 184, no. 10, pp. 589-597, 1996. 
[32] R. Verma, C. Davatzikos, J. Loughead et al., "Quantification of facial expressions using high-dimensional shape transformations," Journal of Neuroscience Methods, vol. 141, no. 1, pp. 61-73, 2005.

[33] C. Alvino, C. Kohler, F. Barrett, R. E. Gur, R. C. Gur, and R. Verma, "Computerized measurement of facial expression of emotions in schizophrenia," Journal of Neuroscience Methods, vol. 163, no. 2, pp. 350-361, 2007.

[34] P. Wang, F. Barrett, E. Martin et al., "Automated video-based facial expression analysis of neuropsychiatric disorders," Journal of Neuroscience Methods, vol. 168, no. 1, pp. 224-238, 2008.

[35] J. Hamm, C. G. Kohler, R. C. Gur, and R. Verma, "Automated facial action coding system for dynamic analysis of facial expressions in neuropsychiatric disorders," Journal of Neuroscience Methods, vol. 200, no. 2, pp. 237-256, 2011.

[36] C. E. Shannon, "A mathematical theory of communication," Bell System Technical Journal, vol. 27, no. 4, Article ID 379423, pp. 623-656, 1948.

[37] W. R. Garner, Uncertainty and Structure as Psychological Concepts, John Wiley \& Sons, New York, NY, USA, 1962.

[38] A. T. Dittman, Interpersonal Messages of Emotion, Springer, New York, NY, USA, 1972.

[39] A. Borst and F. E. Theunissen, "Information theory and neural coding," Nature Neuroscience, vol. 2, no. 11, pp. 947-957, 1999.

[40] M. Costa, A. L. Goldberger, and C. K. Peng, "Multiscale entropy analysis of biological signals," Physical Review E, vol. 71, no. 2, part 1, Article ID 021906, 2005.

[41] J. Jeong, J. C. Gore, and B. S. Peterson, "Mutual information analysis of the EEG in patients with Alzheimer's disease," Clinical Neurophysiology, vol. 112, no. 5, pp. 827-835, 2001.

[42] S. H. Na, S. H. Jin, S. Y. Kim, and B. J. Ham, "EEG in schizophrenic patients: mutual information analysis," Clinical Neurophysiology, vol. 113, no. 12, pp. 1954-1960, 2002.

[43] R. C. Gur, R. Sara, M. Hagendoorn et al., "A method for obtaining 3-dimensional facial expressions and its standardization for use in neurocognitive studies," Journal of Neuroscience Methods, vol. 115, no. 2, pp. 137-143, 2002.

[44] W. R. Garner and H. W. Hake, "The amount of information in absolute judgments," Psychological Review, vol. 58, no. 6, pp. 446-459, 1951.

[45] J. Beirlant, E. J. Dudewicz, L. Gyoerfi, and E. C. Meulen, "Nonparametric entropy estimation: an overview," International Journal of Mathematical and Statistical Sciences, vol. 6, pp. 17-39, 1997.

[46] L. F. Kozachenko and N. N. Leonenko, "Sample estimate of the entropy of a random vector," Problemy Peredachi Informatsii, vol. 23, no. 2, pp. 9-16, 1987.

[47] M. N. Goria, N. N. Leonenko, V. V. Mergel, and P. L. N. Inverardi, "A new class of random vector entropy estimators and its applications in testing statistical hypotheses," Journal of Nonparametric Statistics, vol. 17, no. 3, pp. 277-297, 2005.

[48] R. M. Mnatsakanov, N. Misra, S. Li, and E. J. Harner, "Knnearest neighbor estimators of entropy," Mathematical Methods of Statistics, vol. 17, no. 3, pp. 261-277, 2008.

[49] J. Lin, "Divergence measures based on the Shannon entropy," IEEE Transactions on Information Theory, vol. 37, no. 1, pp. 145151, 1991.

[50] D. M. Endres and J. E. Schindelin, "A new metric for probability distributions," IEEE Transactions on Information Theory, vol. 49, no. 7, pp. 1858-1860, 2003.
[51] S. Kullback and R. A. Leibler, "On Information and Sufficiency," Annals of Mathematical Statistics, vol. 22, no. 1, pp. 79-86, 1951.

[52] B. C. Ho, P. Nopoulos, M. Flaum, S. Arndt, and N. C. Andreasen, "Two-year outcome in first-episode schizophrenia: predictive value of symptoms for quality of life," American Journal of Psychiatry, vol. 155, no. 9, pp. 1196-1201, 1998.

[53] R. E. Gur, C. G. Kohler, J. D. Ragland et al., "Flat affect in schizophrenia: relation to emotion processing and neurocognitive measures," Schizophrenia Bulletin, vol. 32, no. 2, pp. 279287, 2006. 


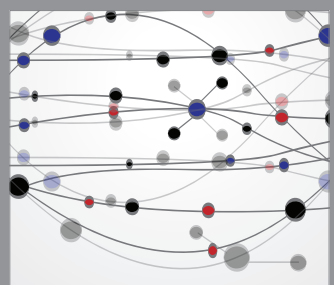

The Scientific World Journal
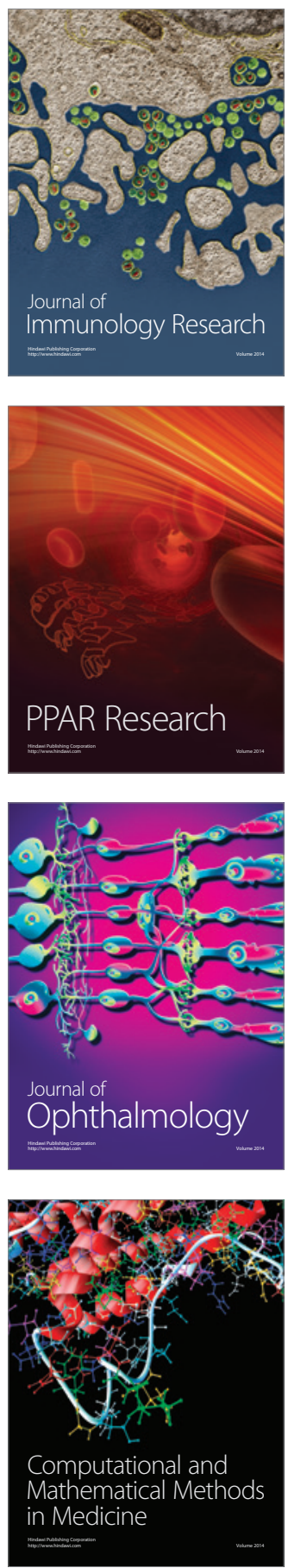

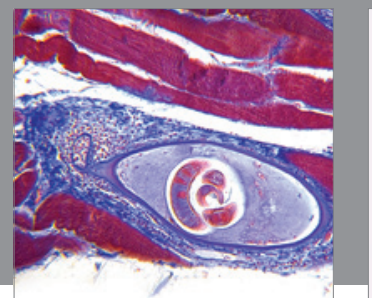

Gastroenterology

Research and Practice
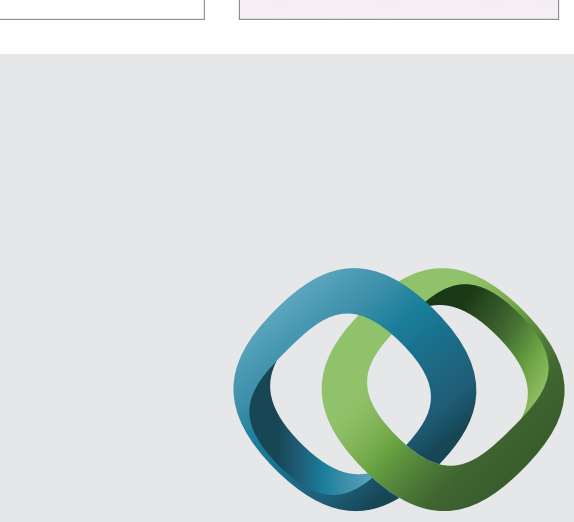

\section{Hindawi}

Submit your manuscripts at

http://www.hindawi.com
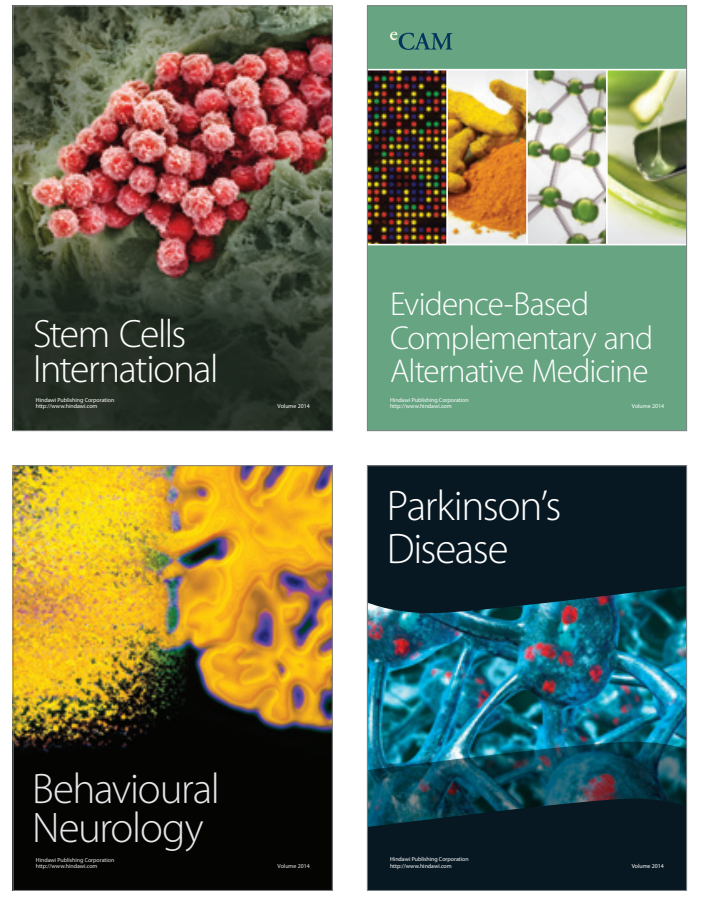
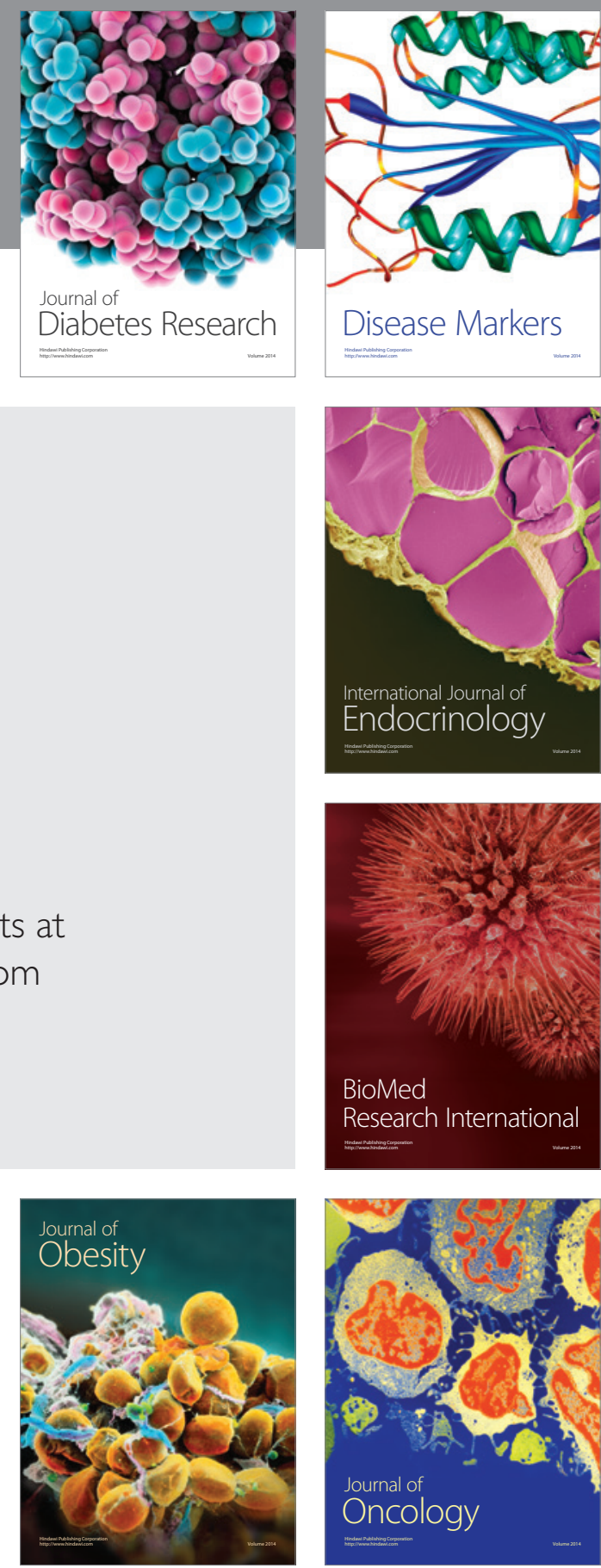

Disease Markers
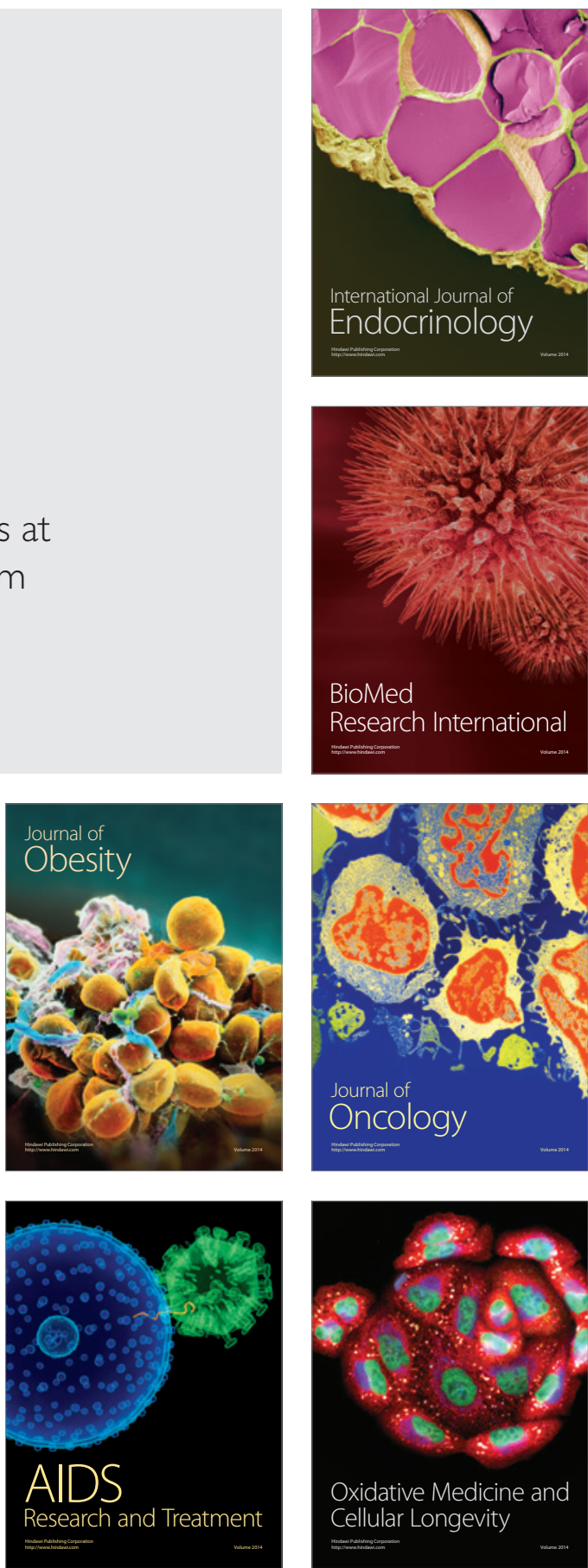